\title{
Using dynamical downscaling to simulate rainfall for East Coast Low events
}

\author{
$\underline{\text { F. Ji }}^{\text {a }}$, J. Evans $^{\text {b }}$ and M. Ekstrom ${ }^{\text {c }}$ \\ ${ }^{a}$ Office of Environment and Heritage, Department of Premier and Cabinet, New South Wales, Australia \\ ${ }^{b}$ Climate Change Research Centre, University of New South Wales, Sydney, Australia \\ ${ }^{c}$ CSIRO Land and Water, Canberra, Australian Capital Territory, Australia \\ Email:fei.ji@environment.nsw.gov.au
}

\begin{abstract}
East Coast Lows (ECLs) are intense low-pressure systems which occur on average several times each year off the eastern coast of Australia, in particular southern Queensland, New South Wales (NSW) and eastern Victoria. East Coast Lows will often intensify rapidly overnight making them one of the more dangerous weather systems to affect and damage the eastern coast of Australia each year. They are also a major source of water for the reservoirs serving coastal communities and ECL events were identified as being responsible for most of the high inflow days in the NSW coastal catchments.

In this study, four East Coast Low events, covering the various ECL categories, are simulated using the Weather Research and Forecasting (WRF) model in order to assess the model's ability to capture the ECL events. By choosing various combinations of two Planetary Boundary Layer (PBL) schemes, two Cumulus (Cu) schemes, three microphysics (mp) schemes and three radiation (ra) schemes, a 36 member multi-physics ensemble is created and evaluated against observations. The uncertainties among the 36 ensemble members are analysed, rainfall probability assessment undertaken and the best estimation of the simulated rainfall are investigated.

The results suggest that the Betts-Miller-Janjic cumulus scheme and the Yonsei University planetary boundary layer scheme can be chosen with some robustness to simulate rainfall pattern and the intensity and position of rainfall centres. Results further indicate that the selection of the cumulus scheme options has the largest impact on model performance during the more intensive rainfall events.

The conclusions from the study can be applied to better estimate rainfall amount and its distribution from dynamical downscaling of climate change projections.
\end{abstract}

Keywords: East Coast Lows (ECLs), Weather Research and Forecasting (WRF), Ensemble, Probability 


\section{INTRODUCTION}

The East coast of Australia from north-east Victoria to south-eastern Queensland is subject to heavy rain, strong winds and large waves resulting from low pressure systems adjacent to the Tasman Sea that develop from a variety of synoptic and mesoscale systems (Speer et al. 2009). These systems, referred to as "East Coast Lows", cause significant damage along the Australian east coast each year. They are also a major source of water for the reservoirs serving coastal communities, and thus are both vital to, and dangerous for, human activities in the area. In this study, four East Coast Lows (ECLs) with varying characteristics are simulated using Weather Forecasting and Research (WRF) model (Skamarock et al, 2008) in order to assess the ability of the model to capture these important events. Initial evaluation of these simulations is presented here. Probability assessment of simulated rainfall and the best rainfall estimation are also investigated.

\section{CASE STUDY PERIODS AND OBSERVATION DATA}

Four different ECL storms were used for the multi-physics ensemble. Events are chosen that represent the most common synoptic situations on the Australian east coast (Speer et al., 2009) accounting for $\sim 85 \%$ of events. Each event is then modelled using a comprehensive 36 member multi-physics ensemble over a two week period such that the evaluation is performed not just on the peak of the storm but on the pre- and poststorm climate as well. In the subsequent analysis, the first day of each simulation is considered as "spin-up" and therefore ignored. The four events occurred on $8^{\text {th }}$ June 2007 (NEWY case), $4^{\text {th }}$ January 2008 (SURFERS case), $4^{\text {th }}$ November 2007 (CTLOW case) and 23 ${ }^{\text {rd }}$ August 2008 (SOLOW case).

Observations used for evaluation come from the gridded dataset prepared as part of the Australian Water Availability Project (AWAP). Details of the creation of this dataset can be found in Jones et al. (2009). This dataset includes precipitation, maximum and minimum temperature, and vapour pressure surfaces obtained by interpolating station measurements. Gridded data from AWAP were re-gridded to the grid specifications of the WRF output data. The 13 days accumulated precipitation for the four ECL events estimated from the AWAP dataset for the period of interest is shown in Figure 1. The characteristics of each storm differ with the first two being large, flood producing storms in winter and summer respectively, while the latter two produce less precipitation. The SURFERS case has three rainfall centres and the major centre is near the Gold Coast. The NEWY case only has one major rainfall centre which is close to Newcastle.

\section{ENSEMBLE DESIGN}

WRF has multiple options for most physics schemes enabling the user to optimise the model for a range of spatial and temporal resolutions and climatologically different geographical regions. By choosing the various combinations of two Planetary Boundary Layer (PBL) schemes (the Yonsei University PBL scheme together with the MM5 similarity theory surface layer scheme and the Mellor-Yamada-Janjic PBL scheme together with the Eta similarity theory surface layer.), two cumulus (cu) schemes (the Kain-Fritsch (KF) scheme and the Betts-Miller-Janjic (BMJ) scheme), three microphysics (mp) schemes (WRF Single

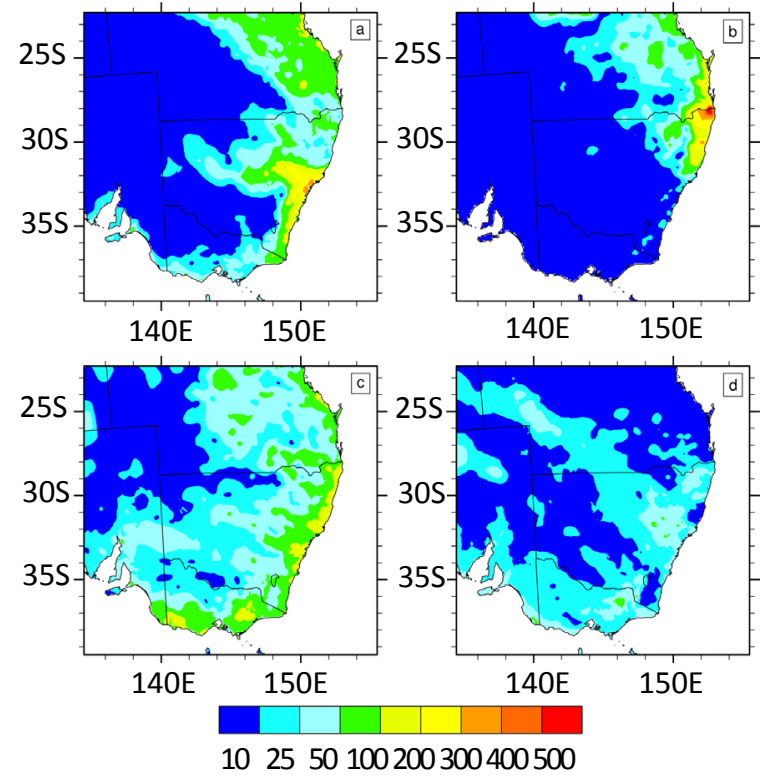

Figure 1. Mapped AWAP rainfall totals (excluding first day of simulation period) for each case study period: a) NEWY, b) SURFRES, c) CTLOW and d) SOLOW

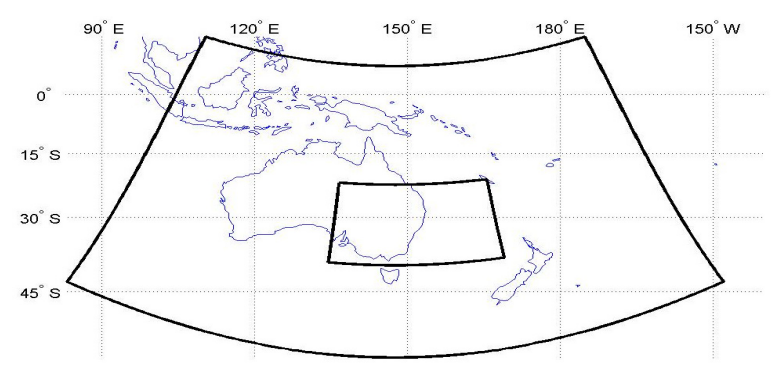

Figure 2. The outer boarder of the outer and inner WRF model domains. The grid cell resolution of the outer/inner domain is $48.9 / 9.8 \mathrm{~km}$. 
Ji et al., Using dynamical downscaling to simulate rainfall in East Coast Low Events

Moment 3-class (WSM3), WRF Single Moment 5-class (WSM5) and the WRF Double Moment 5-class (WDM5)) and three radiation (ra) schemes (Dudhia+RRTM, CAM+CAM, and RRTMG+RRTMG.), a 36 member multi-physics ensemble is created for each case study (Table 1). All the 36 experiments use the Noah land surface scheme (Chen, 2001).

The WRF simulation uses boundary conditions from the ERA-Interim reanalysis with an outer 0.44 degree (about $50 \mathrm{~km}$ ) resolution nest and an inner 0.088 degree (about $10 \mathrm{~km}$ ) resolution nest. The outer domain follows the COordinated Regional climate Downscaling Experiment (CORDEX) Australasia domain (http://wcrp.ipsl.jussieu.fr/RCD CORDEX.html) and the inner domain comprises south-east Australia and the East Coast Low domains (see Figure 2). Both nests used 30 vertical levels spaced closer together in the planetary boundary layer. Boundary conditions and initial starting conditions were derived from ERAInterim using 30 atmospheric levels and 4 soil levels (http://www.ecmwf.int/research/era/do/get/era-interim).

Table 1. Ensemble design, physics options for planetary boundary layer/surface layer physics scheme, cumulus scheme, micro-physics scheme, long wave and short wave radiation schemes.

\begin{tabular}{|c|c|c|c|c|}
\hline $\begin{array}{l}\text { Ensemble } \\
\text { member }\end{array}$ & $\begin{array}{c}\text { Planetary Boundary layer physics / } \\
\text { Surface layer physics }\end{array}$ & $\begin{array}{l}\text { Cumulus } \\
\text { physics }\end{array}$ & Micro-physics & $\begin{array}{l}\text { Shortwave / Longwave } \\
\text { radiation physics }\end{array}$ \\
\hline 1 & YSU / MM5 similarity & KF & WSM 3 class & Dudhia / RRTM \\
\hline 2 & YSU / MM5 similarity & $\mathrm{KF}$ & WSM 3 class & CAM / CAM \\
\hline 3 & YSU / MM5 similarity & KF & WSM 3 class & RRTMG / RRTMG \\
\hline 4 & YSU / MM5 similarity & KF & WSM 5 class & Dudhia / RRTM \\
\hline 5 & YSU / MM5 similarity & KF & WSM 5 class & CAM / CAM \\
\hline 6 & YSU / MM5 similarity & KF & WSM 5 class & RRTMG / RRTMG \\
\hline 7 & YSU / MM5 similarity & KF & WDM 5 class & Dudhia / RRTM \\
\hline 8 & YSU / MM5 similarity & $\mathrm{KF}$ & WDM 5 class & CAM / CAM \\
\hline 9 & YSU / MM5 similarity & KF & WDM 5 class & RRTMG / RRTMG \\
\hline 10 & YSU / MM5 similarity & BMJ & WSM 3 class & Dudhia / RRTM \\
\hline 11 & YSU / MM5 similarity & $\mathrm{BMJ}$ & WSM 3 class & CAM / CAM \\
\hline 12 & YSU / MM5 similarity & BMJ & WSM 3 class & RRTMG / RRTMG \\
\hline 13 & YSU / MM5 similarity & BMJ & WSM 5 class & Dudhia / RRTM \\
\hline 14 & YSU / MM5 similarity & BMJ & WSM 5 class & CAM / CAM \\
\hline 15 & YSU / MM5 similarity & BMJ & WSM 5 class & RRTMG / RRTMG \\
\hline 16 & YSU / MM5 similarity & BMJ & WDM 5 class & Dudhia / RRTM \\
\hline 17 & YSU / MM5 similarity & BMJ & WDM 5 class & CAM / CAM \\
\hline 18 & YSU / MM5 similarity & BMJ & WDM 5 class & RRTMG / RRTMG \\
\hline 19 & MYJ / Eta similarity & KF & WSM 3 class & Dudhia / RRTM \\
\hline 20 & MYJ / Eta similarity & KF & WSM 3 class & CAM / CAM \\
\hline 21 & MYJ / Eta similarity & $\mathrm{KF}$ & WSM 3 class & RRTMG / RRTMG \\
\hline 22 & MYJ / Eta similarity & $\mathrm{KF}$ & WSM 5 class & Dudhia / RRTM \\
\hline 23 & MYJ / Eta similarity & KF & WSM 5 class & CAM / CAM \\
\hline 24 & MYJ / Eta similarity & $\mathrm{KF}$ & WSM 5 class & RRTMG / RRTMG \\
\hline 25 & MYJ / Eta similarity & $\mathrm{KF}$ & WDM 5 class & Dudhia / RRTM \\
\hline 26 & MYJ / Eta similarity & $\mathrm{KF}$ & WDM 5 class & $\mathrm{CAM} / \mathrm{CAM}$ \\
\hline 27 & MYJ / Eta similarity & $\mathrm{KF}$ & WDM 5 class & RRTMG / RRTMG \\
\hline 28 & MYJ / Eta similarity & BMJ & WSM 3 class & Dudhia / RRTM \\
\hline 29 & MYJ / Eta similarity & BMJ & WSM 3 class & CAM / CAM \\
\hline 30 & MYJ / Eta similarity & BMJ & WSM 3 class & RRTMG / RRTMG \\
\hline 31 & MYJ / Eta similarity & BMJ & WSM 5 class & Dudhia / RRTM \\
\hline 32 & MYJ / Eta similarity & BMJ & WSM 5 class & CAM / CAM \\
\hline 33 & MYJ / Eta similarity & $\mathrm{BMJ}$ & WSM 5 class & RRTMG / RRTMG \\
\hline 34 & MYJ / Eta similarity & BMJ & WDM 5 class & Dudhia / RRTM \\
\hline 35 & MYJ / Eta similarity & BMJ & WDM 5 class & CAM / CAM \\
\hline 36 & MYJ / Eta similarity & BMJ & WDM 5 class & RRTMG / RRTMG \\
\hline
\end{tabular}




\section{RESULTS}

\subsection{Temporal characteristics of rainfall event}

The amount of WRF simulated rainfall on each day within the study period is validated against AWAP data, excluding the first simulation day which is considered as the spin-up period. The statistics reported here are calculated over the entire domain each day.

The SURFERS case study is the largest rainfall event of the four cases considered in this study. The AWAP data show that the main rainfall event takes place over day 6 to day 9, peaking at day 7 (Figure 3a). The majority of the WRF simulations display an increase in rainfall around day 7 but the duration is generally longer by at least one day and for a large proportion of simulations rainfall totals peak at day 8 . Furthermore, for the majority of the simulations the rainfall magnitude is underestimated for the early part of the rainfall event (day 6-7) and over-estimated for much of the later part of the event (Figure 3a). The individual bias per ensemble member ranges between -4 and $12 \mathrm{~mm}$. The SOLOW case study is the smallest rainfall event of the four, with a minor peak of rainfall over day 3-5 (Figure 3b). All WRF simulations performed well in terms of the magnitude of the event. Due to the smaller magnitude of this event, biases are smaller for SOLOW with a range between -1 to $3 \mathrm{~mm}$.

For the four cases, the simulated rainfall biases between 36 members are much larger for stronger cases (NEWY and SURFERS) than for weaker cases (CTLOW and SOLOW). Simulations using different physics scheme combination also provide similar results for weaker cases, but much different results for strong cases.
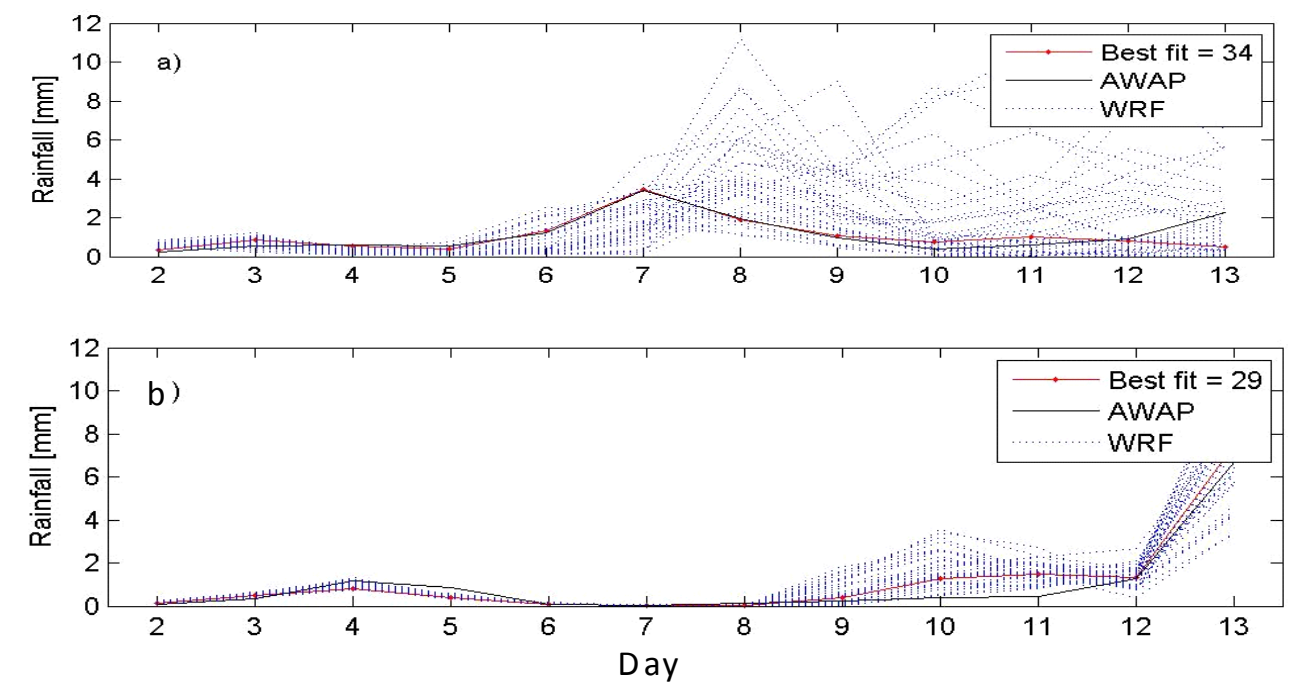

Figure 3. Spatially averaged daily rainfall [mm] simulated by WRF, AWAP daily rainfall totals and closest WRF ensemble member. (a) for SURFERS, b) for SOLOW)

\subsection{Rainfall Probability Assessment}

The ranges of simulated rainfall between ensemble members are small for weak ECL cases but large for strong ECL cases (similar to the results for temporal characteristics of rainfall events). Now we focus on the strong ECL cases (NEWY and SURFERS) to investigate how to improve the simulation accuracy.

Ensemble experiments such as those undertaken in this study make it possible to undertake probability assessment of simulated rainfall. For the high rainfall cases, the rainfall pattern and location of rainfall center are the two important factors to be used to assess simulation accuracy. Based on the observed gridded AWAP rainfall, a $300 \mathrm{~mm}$ of accumulated rainfall was set as the cutoff to determine the boundary of rainfall centre for both NEWY and SURFERS cases.

For the NEWY case, the probability of rainfall over $300 \mathrm{~mm}$ calculated from the 36 ensemble members shows that the maximum probability is about $60 \%$, which is reasonably good from the view of a probability forecast. To investigate if the probability is sensitive to the physical parameterisations, the simulations were

Table 2. The probability of rainfall more than 300mm using different physics scheme for NEWY.

\begin{tabular}{|c|c|c|c|c|c|c|c|c|c|}
\hline \multicolumn{2}{|c|}{ PBL scheme } & \multicolumn{2}{|c|}{ Cu scheme } & \multicolumn{2}{|c|}{ Mp scheme } & \multicolumn{2}{|c|}{ Ra scheme } & \multicolumn{2}{|c|}{ Pbl \& cu schemes } \\
\hline YSU & $>70 \%$ & $\overline{\mathrm{BMJ}}$ & $\sim 75 \%$ & WDM5 & $60-70 \%$ & $\mathrm{CAM}+\mathrm{CMA}$ & $60-70 \%$ & YSU\&BMJ & $>90 \%$ \\
\hline MYJ & $40-50 \%$ & $\mathrm{KF}$ & $\sim 40 \%$ & WSM5 & $60-70 \%$ & RRMTG+RRTMG & $\sim 60 \%$ & YSU\&KF & $45-55 \%$ \\
\hline & & & & WSM3 & $27360 \%$ & Dadhia+RRTM & $\sim 50 \%$ & MYJ\&BMJ & $45-55 \%$ \\
\hline
\end{tabular}


grouped according to the physics schemes including pbl \& land surface scheme, cu scheme, mp scheme and ra schemes respectively. The results are summarized in Table 2.

Based on the above results, all of the experiments which use the scheme combinations of YSU\&MM5 pbl \& surface layer scheme, BMJ cu scheme and two mp schemes (WSM5, WDM5) and two ra schemes $(\mathrm{CAM}+\mathrm{CAM}$ and RRTMG+RRTMG) were used to calculate the probability of rainfall over $300 \mathrm{~mm}, 200 \mathrm{~mm}$ and $100 \mathrm{~mm}$, respectively. The results are better than those calculated from groups using other physics scheme combinations, and the higher probabilities of rainfall for over $100 \mathrm{~mm}, 200 \mathrm{~mm}$ and $300 \mathrm{~mm}$ match the rainfall pattern and the location of the rainfall centre reasonably well (see Figure 4). The maximum probability of rainfall is more sensitive to $\mathrm{cu}$ and $\mathrm{pbl} \&$ surface layer schemes than $\mathrm{mp}$ and ra schemes.

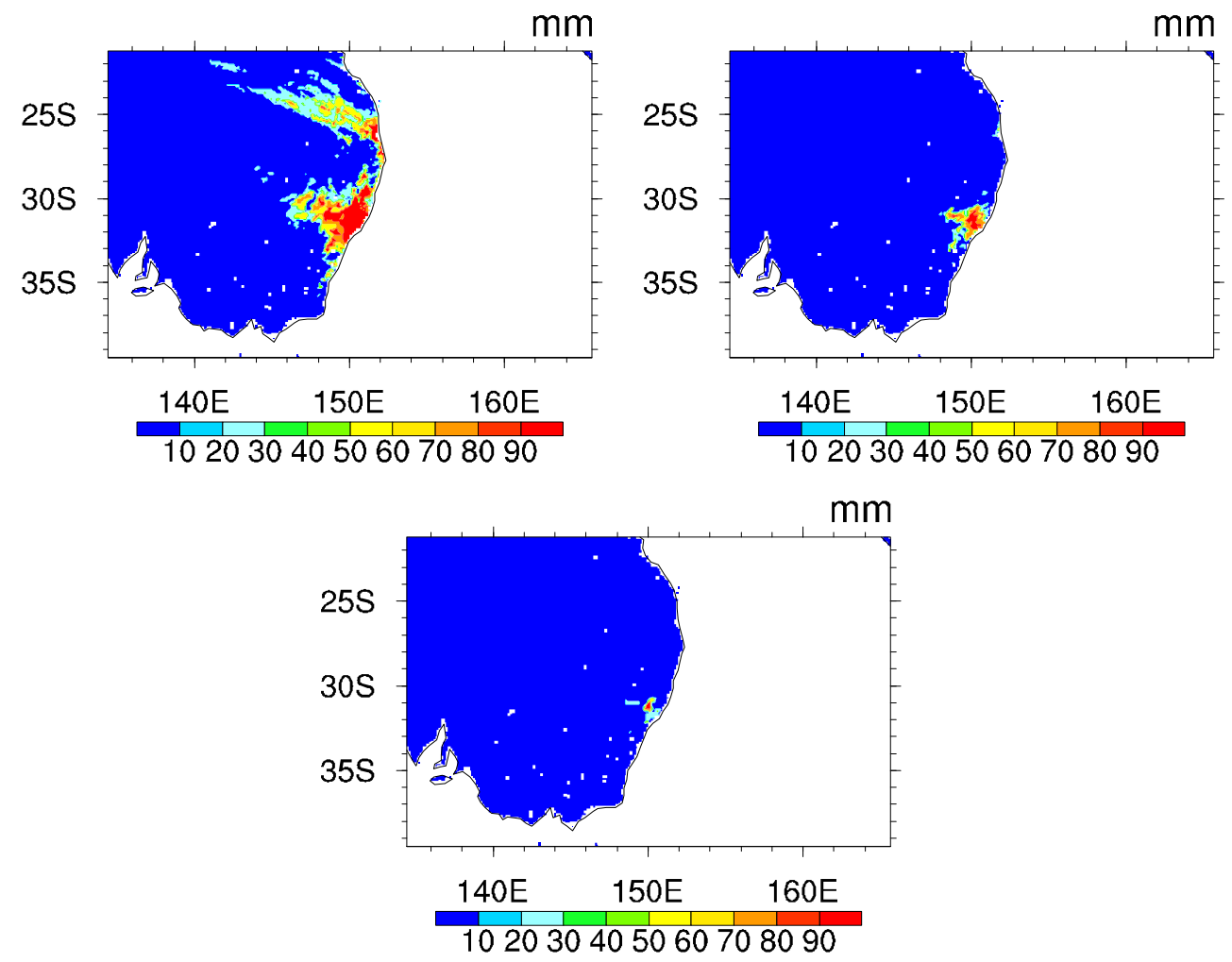

Figure 4. Probabilities for rainfall over $100 \mathrm{~mm}, 200 \mathrm{~mm}$ and $300 \mathrm{~mm}$, respectively, for the NEWY case.

Table 3. The probability of rainfall more than 300mm using different physics scheme for SURFRES.

\begin{tabular}{cccccccccc}
\hline \multicolumn{2}{c}{ PBL scheme } & \multicolumn{2}{c}{ Cu scheme } & \multicolumn{2}{c}{ Mp scheme } & \multicolumn{2}{c}{ Ra scheme } & \multicolumn{2}{c}{ Pbl \& cu schemes } \\
\hline YSU & $40-50 \%$ & BMJ & $50-60 \%$ & WDM5 & $40-50 \%$ & CAM+CMA & $35-45 \%$ & YSU\&BMJ & $60-70 \%$ \\
\hline MYJ & $20-30 \%$ & KF & $10-20 \%$ & WSM5 & $40-50 \%$ & RRMTG+RRTMG & $40-50 \%$ & YSU\&KF & $20-30 \%$ \\
\hline & & & WSM3 & $40-50 \%$ & Dadhia+RRTM & $35-45 \%$ & MYJ\&BMJ & $50-60 \%$ \\
\hline & & & & & & MYJ\&KF & $20-30 \%$ \\
\hline
\end{tabular}

The same analyses were undertaken for SURFERS case. The results are summarized in the table 3 . We also used all of the experiments with the scheme combinations of YSU\&MM5 pbl \& surface layer scheme, BMJ cu scheme and two mp schemes (WSM5, WDM5) and two ra schemes (CAM+CAM and RRTMG+RRTMG) to calculate the probability of rainfall over $300 \mathrm{~mm}, 200 \mathrm{~mm}$ and $100 \mathrm{~mm}$, respectively. The results are better than those calculated from groups using other physics scheme combinations as well. Furthermore, it generally reproduces the observed rainfall pattern and the three rainfall centres as shown on Figure 5. 


\subsection{Best estimation of rainfall from ensemble members}

For the NEWY case, the rainfall pattern of ensemble mean from the 36 members matches the observation reasonably well; but the maximum simulated rainfall is about $325 \mathrm{~mm}$ which is lower than the $440 \mathrm{~mm}$ observed.

The simulated rainfall at the centre estimated by the ensemble mean from the group using YSU\&MM5 scheme is about $375 \mathrm{~mm}$, much better than $280 \mathrm{~mm}$, from the group using MYJ\&Eta scheme; using BMJ cu scheme is $380 \mathrm{~mm}$, much better than about $270 \mathrm{~mm}$ when using the KF cu scheme; is about $375 \mathrm{~mm}$ when using WDM5 mp scheme, better than about 300mm using the other two mp schemes. The patterns are similar
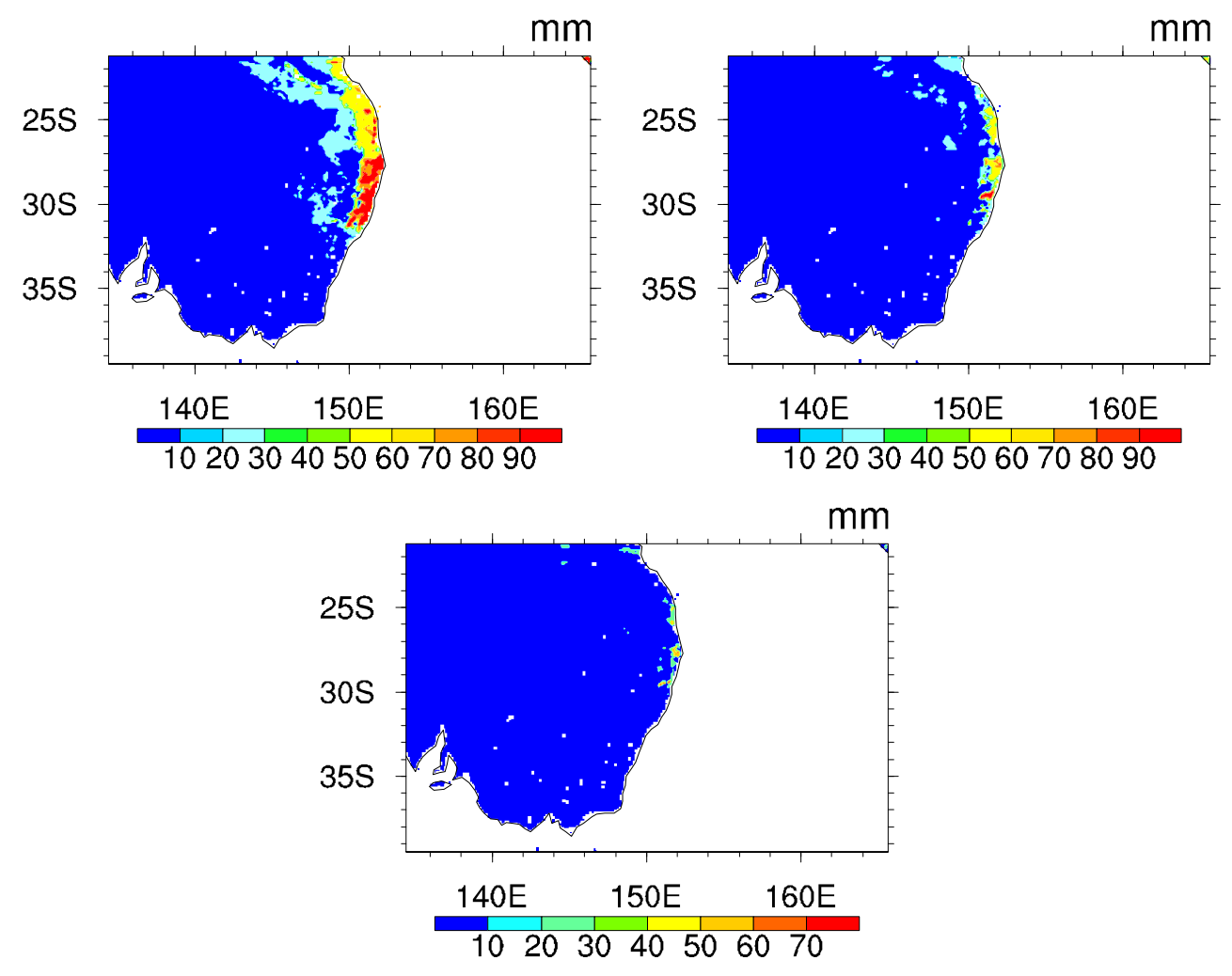

Figure 5. Probabilities for rainfall over $100 \mathrm{~mm}, 200 \mathrm{~mm}$ and $300 \mathrm{~mm}$, respectively, for the SURFERS case.

when using the three ra schemes but the value from the group using CAM+CAM scheme is $350 \mathrm{~mm}$, slight better than that using the other two schemes.

We further combined the two pbl \& surface layer schemes with the two cu schemes, the ensemble mean using YSU\&MM5 scheme combined with BMJ cu scheme is about 440mm, better than using the other three scheme combinations. We also used results from all of the experiments using the scheme combinations of YSU\&MM5 scheme, BMJ cu scheme and two mp schemes (WSM5, WDM5) and two ra schemes $(\mathrm{CAM}+\mathrm{CAM}$ and RRTMG+RRTMG) to calculate mean rainfall, and the results are better than those calculated from groups using other physics scheme combinations ( Figure 6).
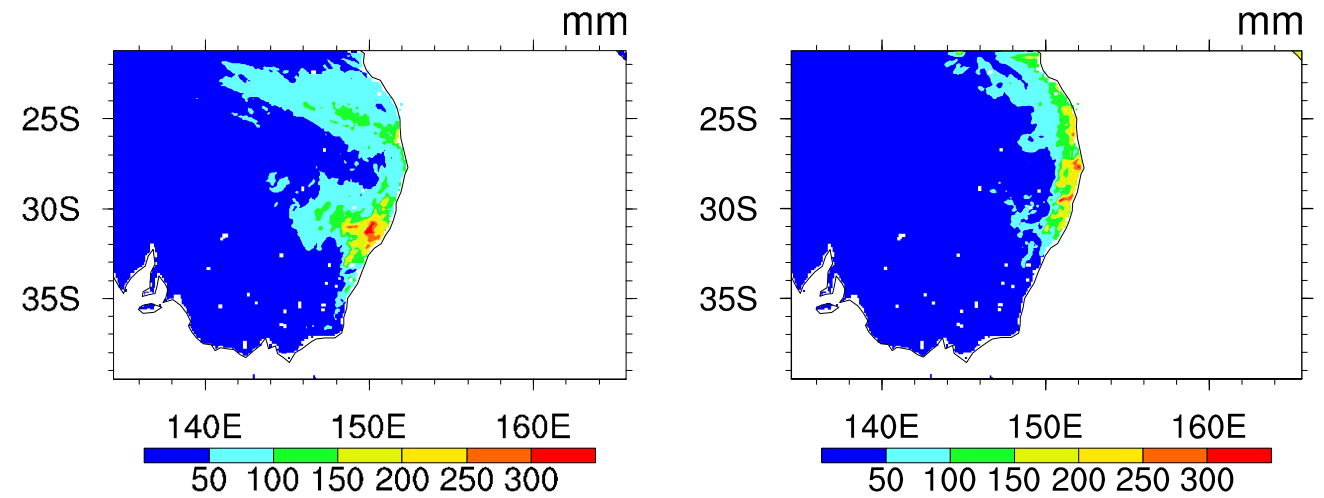

Figure 6. The best estimated rainfalls for NEWY (left) and SURFERS (right). 
For the SURFERS case, the rainfall pattern of ensemble means from the 36 members generally match the observation, but the maximum rainfall is about $300 \mathrm{~mm}$, much lower than the $700 \mathrm{~mm}$ observed.

The rainfall centre of the ensemble mean from the group using YSU\&MM5 pbl and surface layer scheme is similar to that from the group using MYJ\&Eta pbl and surface layer scheme, but the latter underestimated the south rainfall centre and overestimated the north rainfall and the first combination simulated a better pattern than the second. The rainfall amount when using BMJ cu scheme is $350 \mathrm{~mm}$, much better than using the KF cu scheme (about 220mm); using WDM5 mp scheme is $350 \mathrm{~m}$, better than using the other two mp schemes (about $300 \mathrm{~mm}$ ). The simulated rainfall patterns are similar when using the three ra schemes but the value from the group using RRTMG+RRTMG scheme is about $350 \mathrm{~mm}$ which is better than using the CAM+CAM scheme (300mm) and Dadhia+RRTM scheme (250mm).

We further combined the two pbl\& surface layer schemes with the two cu schemes. The ensemble mean using the two pbl and surface layer schemes combined with BMJ cu scheme are better than other two using two pbl and surface layer schemes combining with KF cu scheme. The experiments using MYJ \& Eta pbl and surface layer scheme combined with BMJ cu scheme overestimated the north rainfall centre and underestimated the south rainfall centre. We also used simulations from all of the experiments using the scheme combinations of YSU\&MM5 pbl \& surface layer scheme, BMJ cu scheme and two mp schemes (WSM5, WDM5) and two ra schemes (CAM\&CAM and RRTMG\&RRTMG) to calculate mean rainfall, and the results are better than other combinations which can reproduce the three rainfall centres quite well but with simulated rainfall amounts much lower than the observations (see Figure 6).

\section{CONCLUSIONS}

This study evaluated the ability of a collection of WRF simulations to reproduce four East Coast Low events near the eastern coast of Australia. A 36 member multi-physics ensemble was used to explore the sensitivity of the simulations to various physical parameterisations. AWAP data was used to validate the performance of the multi-physics ensemble members. The differences between the members of the multi-physics ensemble are much smaller for relatively weak weather systems than for strong or extreme weather systems.

The probabilities of simulated rainfall and the mean rainfall are more sensitive to cu and pbl \& surface layers schemes than $\mathrm{mp}$ and ra schemes. BMJ cu scheme performed much better than KF scheme; YSU\&MM5 scheme performed better than MYJ\&Eta scheme; WSD5, WSM5 mp schemes and RRTMG+RRTMG, CAM+CAM ra schemes performed slightly better than WSM3 and Dudhia+RRTM respectively.

The best results are obtained from the model runs using the physics scheme combinations of YSU\&MM5 scheme, BMJ cu scheme, two mp schemes (WSM5 and WDM5) and two ra schemes (CAM+CAM, RRTMG+RRTMG).

\section{ACKNOWLEDGMENTS}

This work is made possible by funding from the NSW Environmental Trust for the ESCCI-ECL project, the NSW Office of Environment and Heritage backed NSW/ACT Regional Climate Modelling Project (NARCliM), and the Australian Research Council as part of the Discovery Project DP0772665. Thanks to the Australian Climate Change Science Program (ACCSP) and the South Eastern Australian Climate Initiative (SEACI) for funding the CSIRO contribution to this study. This research was undertaken on the NCI National Facility in Canberra, Australia, which is supported by the Australian Commonwealth Government.

\section{REFERENCES}

Chen, F., and Dudhia, J. (2001). Coupling an advanced land-surface/ hydrology model with the Penn State/ NCAR MM5 modeling system. Part I: Model description and implementation. Mon. Wea. Rev., 129, 569585.

Jones, D., Wang, W. and Fawcett, R. (2009). High-quality spatial climate data-sets for Australia. Australian Meteorological and Oceanographic Journal, 58 , 233-248

Skamarock,W., Klemp, J. B., Dudhia, J., et al (2008). A Description of the Advanced Research WRF Version 3, NCAR TECHNICAL NOTE, NCAR/TN-475+STR

Speer, M., Wiles, P. and Pepler, A. (2009). Low pressure systems off the New South Wales coast and associated hazardous weather: establishment of a database. Australian Meteorological and Oceanographic Journal, 58, 29-39 